\title{
The Effect of Different Types of Mechanical Circulatory Support on Mortality of Patients after Adult Cardiac Surgery: A Systematic Review and Meta-Analysis
}

\author{
Zhiyuan Guan, PhD, ${ }^{1}$ Xiaoqing Guan, $\mathrm{PhD},{ }^{2}$ Kaiyun Gu, PhD,${ }^{1}$ Yanqi Li, MD,${ }^{3}$ Jin Lin, MD,${ }^{1}$ \\ Wenjun Zhou, MD, ${ }^{1}$ Ming Xu, PhD,${ }^{4}$ Chunli Song, PhD,${ }^{5}$ Zhe Zhang, MD, ${ }^{1}$ Feng $\mathrm{Wan}^{6}$ \\ ${ }^{1}$ Peking University Third Hospital, Haidian District, Beijing, China; ${ }^{2}$ Peking University, Beijing, China; ${ }^{3}$ Beijing University \\ of Technology, Beijing, China; ${ }^{4}$ Department of Cardiology, Peking University Third Hospital, NHC Key Laboratory of \\ Cardiovascular Molecular Biology and Regulatory Peptides, Beijing, China; ${ }^{5}$ Department of Orthopedics, Peking University \\ Third Hospital, Beijing, China; ${ }^{6}$ Shanghai East Hospital, Tongji University, Pudong District, Shanghai, China
}

\section{ABSTRACT}

Objectives: Sample size may limit the ability of individual studies to detect differences in clinical outcomes between extracorporeal membrane oxygenation (ECMO) alone and ECMO plus intra-aortic balloon pump (IABP) after adult cardiac surgery. Therefore, we undertook a meta-analysis of the best evidence available on the comparison of clinical outcomes of ECMO alone and ECMO plus IABP after adult cardiac surgery.

Methods: PubMed, EMBASE, Web of Science, and Cochrane Center Registry of Controlled Trials were searched for studies comparing the use of ECMO alone and ECMO plus IABP after adult cardiac surgery. A meta-analysis and a sensitivity analysis were conducted.

Results: Among the 472 screened articles, 24 studies (1302 cases of ECMO plus IABP and 1603 cases of ECMO) were included. A significant relationship between patient risk profile and benefits from IABP plus ECMO was found in terms of the 30-day mortality (odds ratio [OR] 0.75; 95\% confidence interval [CI] 0.62 to $0.91 ; P=.004)$ with postcardiotomy shock (PCS). However, ECMO alone was associated with lower in-hospital mortality (OR 1.75; 95\% CI 1.06 to 3.01; $Z=2.19 ; P=.03$ ) compared with ECMO plus IABP without PCS.

Conclusions: Pooled data show that patients receiving IABP plus ECMO with PCS have lower 30-day mortality than those receiving ECMO also, which in turn show higher 30-day mortality in patients with IABP plus ECMO without PCS. Further randomized studies are warranted to corroborate these observational data.

\section{INTRODUCTION}

Severe postcardiotomy myocardial dysfunction after cardiac surgery is a leading cause of mortality, and the utilization

Received March 24, 2020; accepted March 26, 2020.

Correspondence: Zhe Zhang, 49 North Garden Rd., Haidian District, Beijing 100191, China (e-mail: zhangzhe@bjmu.edu.cn); Chunli Song, 49 North Garden Rd., Haidian District, Beijing 100191, China (e-mail: scbl@bjmu.edu.cn). rates of extracorporeal membrane oxygenation (ECMO) and intra-aortic balloon pump (IABP) can be $3 \%$ to $5 \%$ in the patients with postcardiotomy shock (PCS) after cardiac surgery [Guttendorf 2014; Ko 2002]. Traditionally, the main life-threatening complications of cardiac surgery fall into 2 categories, PCS and postcardiotomy failure (PCF) [Jaski 2010; Magovern 1994], both of which show higher mortality $(>60 \%)$ [Committee for Scientific Affairs 2011]. PCS also remains the leading cause of death in patients with acute myocardial infarction (AMI), myocarditis, and graft failure after heart transplantation. In addition to pharmacologic measures, treatment with mechanical circulatory support can

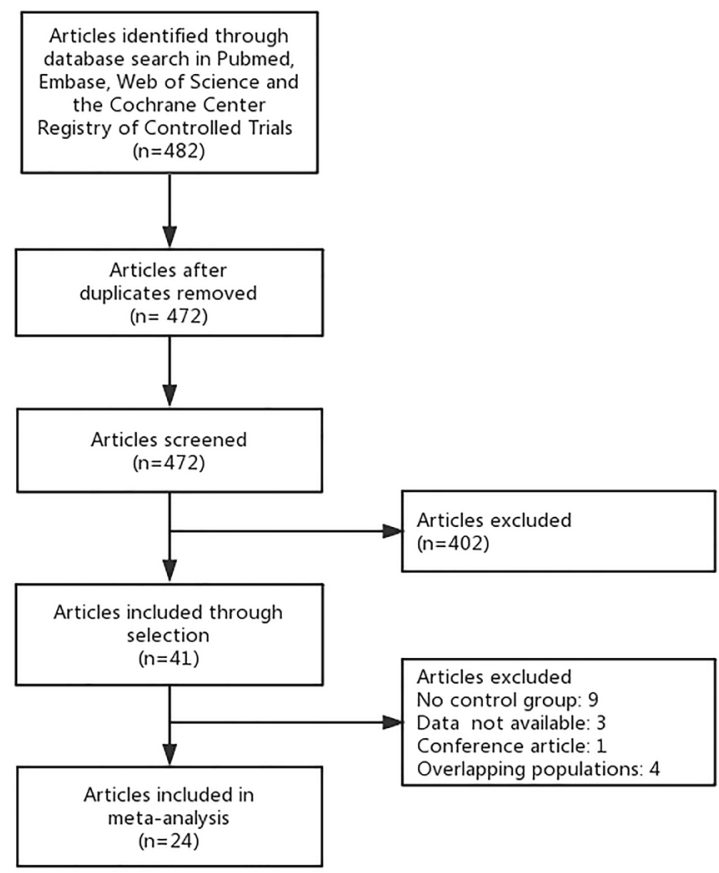

Figure 1. Search strategy. 
Table 1. Quality Analysis of 24 Studies*

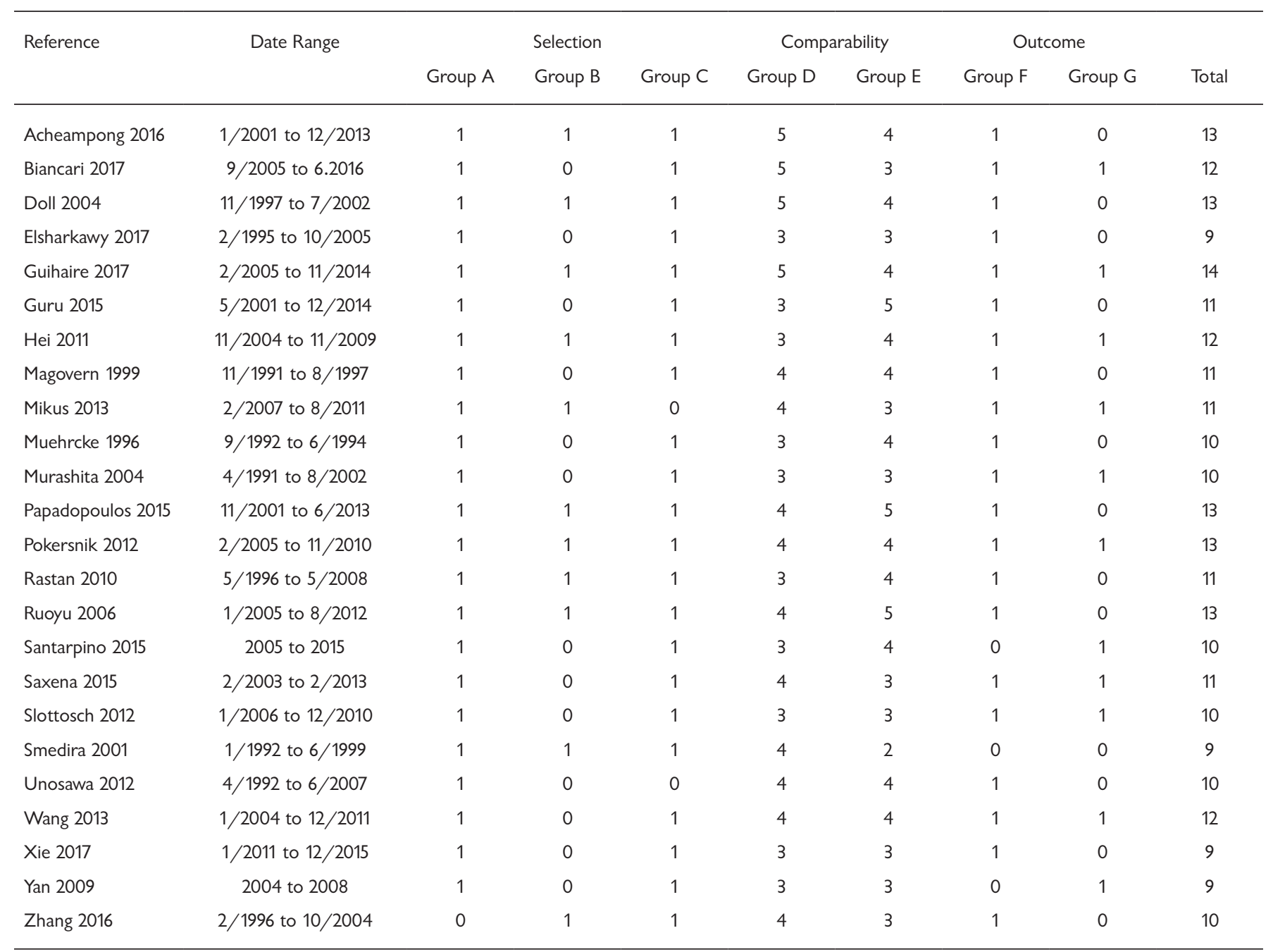

*Group A: Assignment for treatment-any criteria reported (if yes, score 1)? Group B: How representative was the reference group (VA-ECMO) in comparison to the general population after cardiac surgery? (If yes, score 1; no score if the patients were selected or selection of group was not described). Group C: How representative was the treatment group ( IABP plus ECMO) in comparison to the general population after cardiac surgery? (If drawn from the same community as the reference group, score 1; no score if drawn from a different source or selection of group was not described.) Comparability variables: (1) age; (2) sex; (3) hypertension; (4) diabetes; (5) ejection fraction; (6) 3-vessel disease; (7) left main stem disease; (8) urgent/emergency operation; (9) viability studies; (10) surgeon or hospital volume. Group D: Groups comparable for 1, 2, 3, 4, 5. (If yes, score 1 for each; no score was assigned if the 2 groups differed.) Group E: Groups comparable for 6, 7, 8, 9, 10. (If yes, score 1 for each; no score was assigned if the 2 groups differed.) Group F: Clearly defined outcome of interest (if yes, score 1). Group G: Follow-up (score 1 if described.)

be considered, especially in more severe forms of circulatory failure [Hoy 2000]. The aim of mechanical circulatory support, for example ECMO and IABP, is to unload the failing heart ventricle and provide temporary circulatory support for vital organs.

ECMO, also called extracorporeal life support (ELS), is regarded as a modified way to treat critically ill patients with respiratory and cardiovascular failure, especially after cardiac surgery [Gray 2015; Cashen 2015]. However, an increase of left ventricle afterload, elevated left ventricular end-diastolic pressure, acute pulmonary edema, and increased myocardial oxygen consumption are detrimental side effects of ECMO [Chung 2017; Burkhoff 2015]. Nevertheless, it is still unclear whether ECMO effectively increases the survival rate [Meani 2017].

IABP is no longer recommended as a routine therapy for patients after cardiac surgery [Thiele 2013], and the role of IABP in providing additional circulatory support and left ventricular decompression in patients with concomitant ECMO is controversial [Cheng 2015]. IABP could theoretically reduce aortic volume through a vacuum-like effect, improve coronary blood flow and cardiac output, and reduce heart rate and pulmonary capillary wedge pressure [Altayyar 2014]. In 


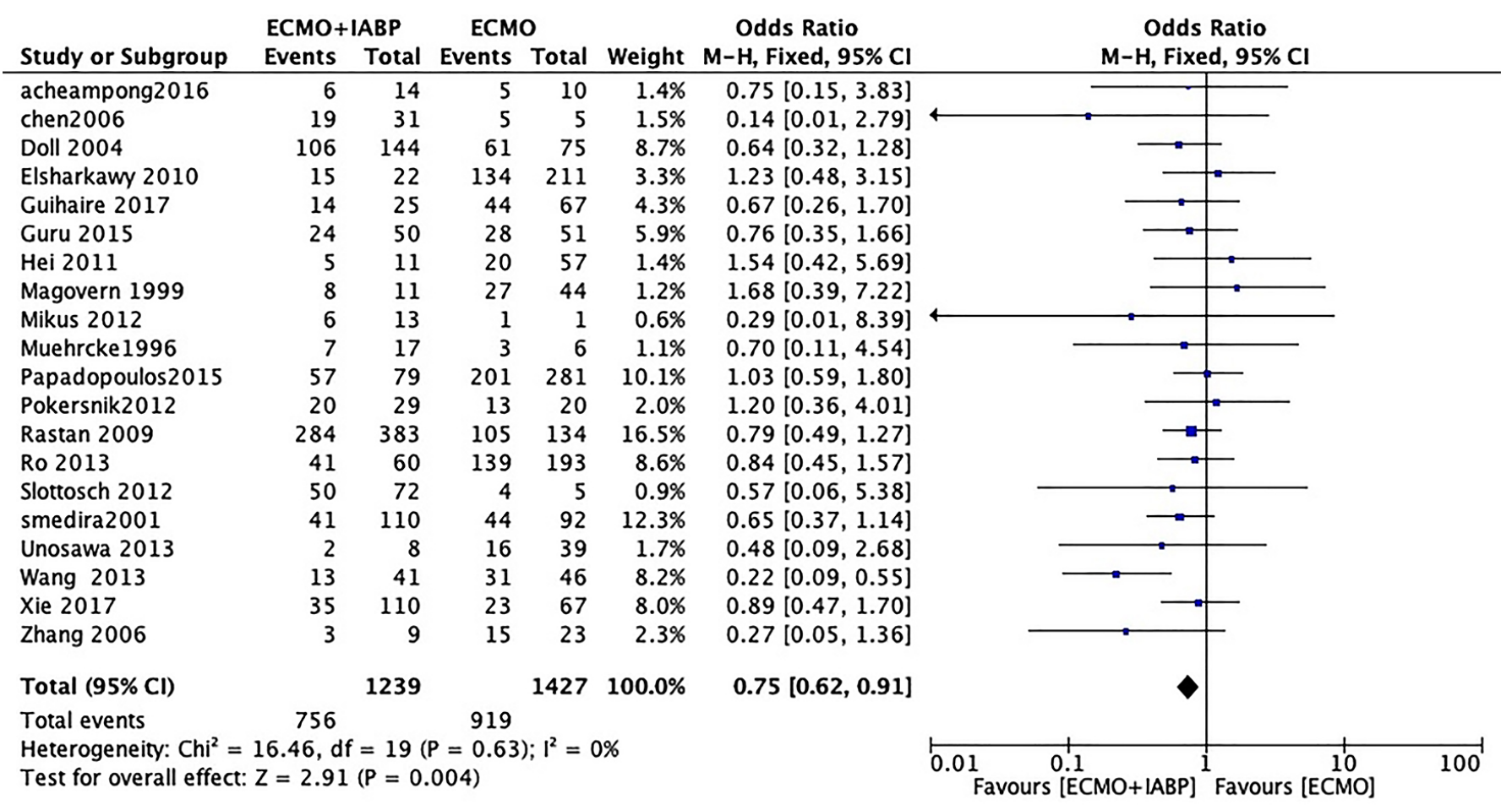

Figure 2. In-hospital mortality between ECMO plus IABP and ECMO alone in cardiac surgery with PCS.

light of the multiple recent studies with contrasting results [Thiele 2012; Aso 2016; Mascio 2014], we sought to systematically review the available evidence of the role of concomitant IABP for patients with cardiogenic shock. Studies of IABP as a second conduit have focused on all-cause death and have shown better survival rates for ECMO alone [Vallabhajosyula 2018; Mascio 2014]. Therefore, our primary hypothesis that use of concomitant IABP is associated with higher short-term mortality in patients after cardiac surgery with ECMO.

\section{METHODS}

This systematic review and meta-analysis was conducted according to the guidelines of Preferred Reporting Items for Systematic Reviews and Meta-analysis (PRISMA) and Metaanalysis of Observational Studies in Epidemiology (MOOSE) [Liberati 2009; Stroup 2000].

\section{Search Strategy and Definition}

A medical librarian developed searches to identify studies that compared clinical outcomes between IABP and ECMO. PubMed, EMBASE, Web of Science, and Cochrane Center Registry of Controlled Trials were searched for studies published from January 1990 to January 2018. Searches used subject headings and keywords for the following terms: "intra-aortic balloon pump," "extracorporeal membrane oxygenation," "cardiac surgery," "postcardiotomy shock," "cardiopulmonary bypass," "postcardiotomy failure," and "circulatory assist devices."
To be eligible for inclusion in the meta-analysis, trials had to conform to the following criteria: observational studies comparing ECMO and IABP as main technique for cardiac surgery including a majority of operations (coronary artery bypass graft, mitral, aortic valve repair, combination surgery, dissection, etc). Animal studies, reviews, and urgent/emergent cases were excluded. Studies that did not have any of the desired outcome measures or included patients treated by other modalities were excluded; those with incomplete data were excluded. Studies that included interventions other than IABP versus ECMO were excluded (Figure 1).

Patients were considered to be candidates for ECMO if satisfactory systemic perfusion could not be maintained despite high-dose inotropic agents, IABP, or both. The fundamental criteria for ECMO support were as follows: systolic blood pressure $<80 \mathrm{mmHg}$, left atrial pressure $>20 \mathrm{mmHg}$, cardiac index of $1.8 \mathrm{~L} / \mathrm{min} / \mathrm{m}^{2}$, and drug-resistant fetal arrhythmia. PCS was defined as heart failure that either resulted in an inability to wean from cardiopulmonary bypass or that occurred in the immediate postoperative period, accounting for the most common indication for mechanical circulatory support (MCS).

\section{Ethics Approval}

As a meta-analysis, no patients were involved the study, making ethics approval unnecessary.

\section{Data Extractions and Quality Assessment}

Three reviewers (Z. Guan; K. Gu; J. Lin) independently extracted the following data from each study: first author, year of publication, trial characteristics, study design, inclusion 


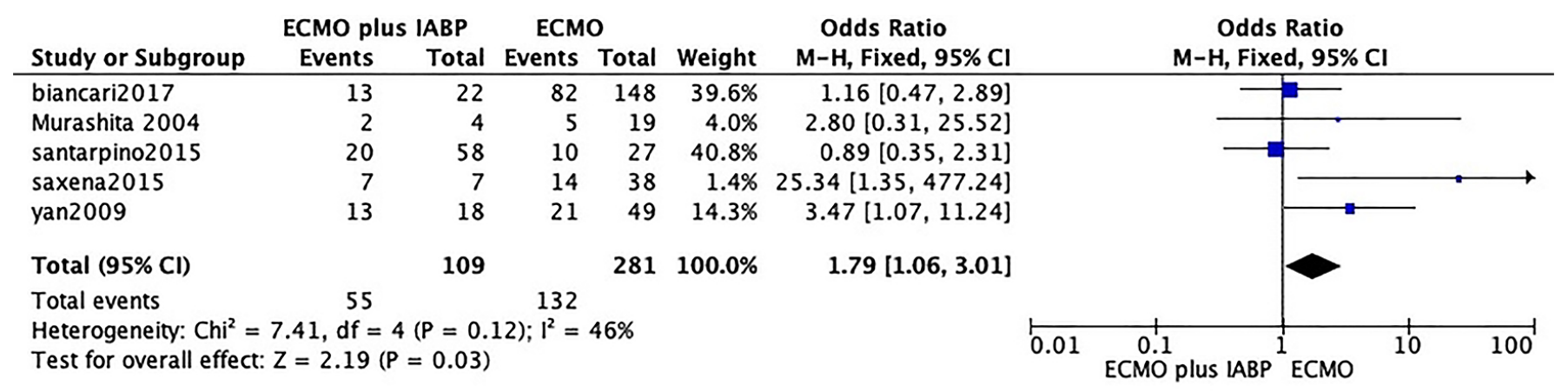

Figure 3. In-hospital mortality between ECMO plus IABP and ECMO alone in cardiac surgery without PCS.

and exclusion criteria, graft type, and clinical outcome (Table 1). The following variables were included: study demographics (sample size, design, and country), patient demographics and comorbidities (age, sex, diabetes status, ejection fraction, chronic obstructive pulmonary disease status). The modified Newcastle-Ottawa scale, summarized in Table 1, is used in our meta-analysis with a quality assessment score. We define a study score of $>6$ as high quality. The quality of all studies was evaluated by 2 independent researchers (W. Zhou; J. Lin).

\section{Description of Outcomes}

The operative mortality was defined as in-hospital mortality within 30 days after cardiac surgery of ECMO alone or ECMO plus IABP [Acheampong 2016].

\section{Statistical Analysis}

The efficacy of ECMO alone and ECMO plus IABP was compared directly by pooling data from the included studies using meta and metaphor packages in R (version 3.5.3, R Project; R Foundation for Statistical Computing, Vienna, Austria) [Viechtbauer 2010]. We pooled the clinical outcomes using odds ratios (ORs) with 95\% confidence intervals (CIs). ORs were used as the common measure for dichotomous data. The random-effects model was used because of the variation among studies due to patients undergoing operations in different centers with varying risk profiles, as well as different selection criteria for each surgical technique. We evaluated heterogeneity by focusing on ECMO alone and ECMO plus IABP after cardiac surgery and a quality score $>6$; heterogeneity was reported as low $\left(I^{2}=0 \%\right.$ to $\left.25 \%\right)$, moderate $\left(I^{2}=26 \%\right.$ to $\left.50 \%\right)$, or high $\left(I^{2}>\right.$ $50 \%)$, consistent with guidelines. Publication bias was assessed visually by funnel plot and quantitatively by the Egger test [Higgins 2003]. We calculated pooled ORs using the MantelHaenszel method, with weight assigned to each included study adjusted to include a measure of variation in the effects reported between studies. Statistical significance was assumed for $P<.05$.

\section{RESULTS}

\section{General Characteristics of the Included Studies}

Table 2 is presents the baseline characteristics of the metaanalysis. All studies were retrospective observational studies, and 8 studies conducted by the Extracorporeal Life Supporting Organization Registry Center, with nonoverlapping patients [Acheampong 2016; Guihaire 2017; Biancari 2017; Toshifumi 2015; Saxena 2015; Santarpino 2015; Papadopoulos 2015; Guru 2015; Kyun 2014; Wang 2013; Satoshi 2013; Ingo 2013; Pokersnik 2012; Feilong 2011; Elsharkawy 2010; Ardawan 2010; Doll 2004; Smedira 2001; Magovern 1999; Muehrcke 1996; Mikus 2013; Xie 2017; Xiaolei 2010; Ruoyu 2006].

Among the 472 screened articles, a total of 24 studies (2905 patients; $1302 \mathrm{ECMO}$ plus IABP and $1603 \mathrm{ECMO}$ alone) met the inclusion criteria (Table 2). Eight studies were in the United States, 5 in Germany, 3 in China, 3 in Japan, 2 in France, 1 in "Europe," and 1 each from Korea and Italy. All observational studies included were matched or adjusted and were of high quality and low risk of bias. The number of patients in the individual studies ranged from 4 to 340 in the ECMO plus IABP group and 1 to 211 in the ECMO alone group. The mean age of the population was 58.70 years; $71.3 \%$ were men; and the average time on ECMO was 5.01 days. The overall in-hospital mortality was about $66.67 \%$. Based on patients with cardiac surgery with or without PCS, the mortality was $62.35 \%$ with PCS and $37.39 \%$ without PCS.

\section{In-Hospital Mortality}

In-hospital mortality was $61.02 \%$ (756 of 1239) with IABP plus ECMO versus $64.40 \%$ (919 of 1427) with ECMO alone (incidence rate ratio $0.75 ; 95 \%$ CI 0.62 to $0.91, P<.05$ ) (Figure 2 ) in the group with cardiac surgery with PCS. In patients having cardiac surgery without PCS, the in-hospital mortality was significantly different for ECMO with and without IABP: $50.46 \%$ (55 of 109 ) versus $46.98 \%$ (132 of 281$)$; (OR $1.79 ; 95 \%$ CI 1.06 to $3.01 ; Z=2.19 ; P=.03)$. Heterogeneity between studies was moderate in these studies $\left(\chi^{2}=7.59\right.$; $\left.P=.16 ; I^{2}=34 \%\right)$, and the fixed model was suggested in subgroup analysis (Figure 3). A funnel plot shows low publication bias $\left(\chi^{2} 22.21 ; P=.27 ; I^{2}=14 \%\right)$ (Figure 4$)$, and the Egger test intercept was -1.23 to $0.13, P=.21$.

\section{DISCUSSION}

We conducted a meta-analysis with 2905 patients treated with IABP plus ECMO or ECMO alone for PCS and other 
Table 2. Baseline between ECMO plus IABP and ECMO Alone

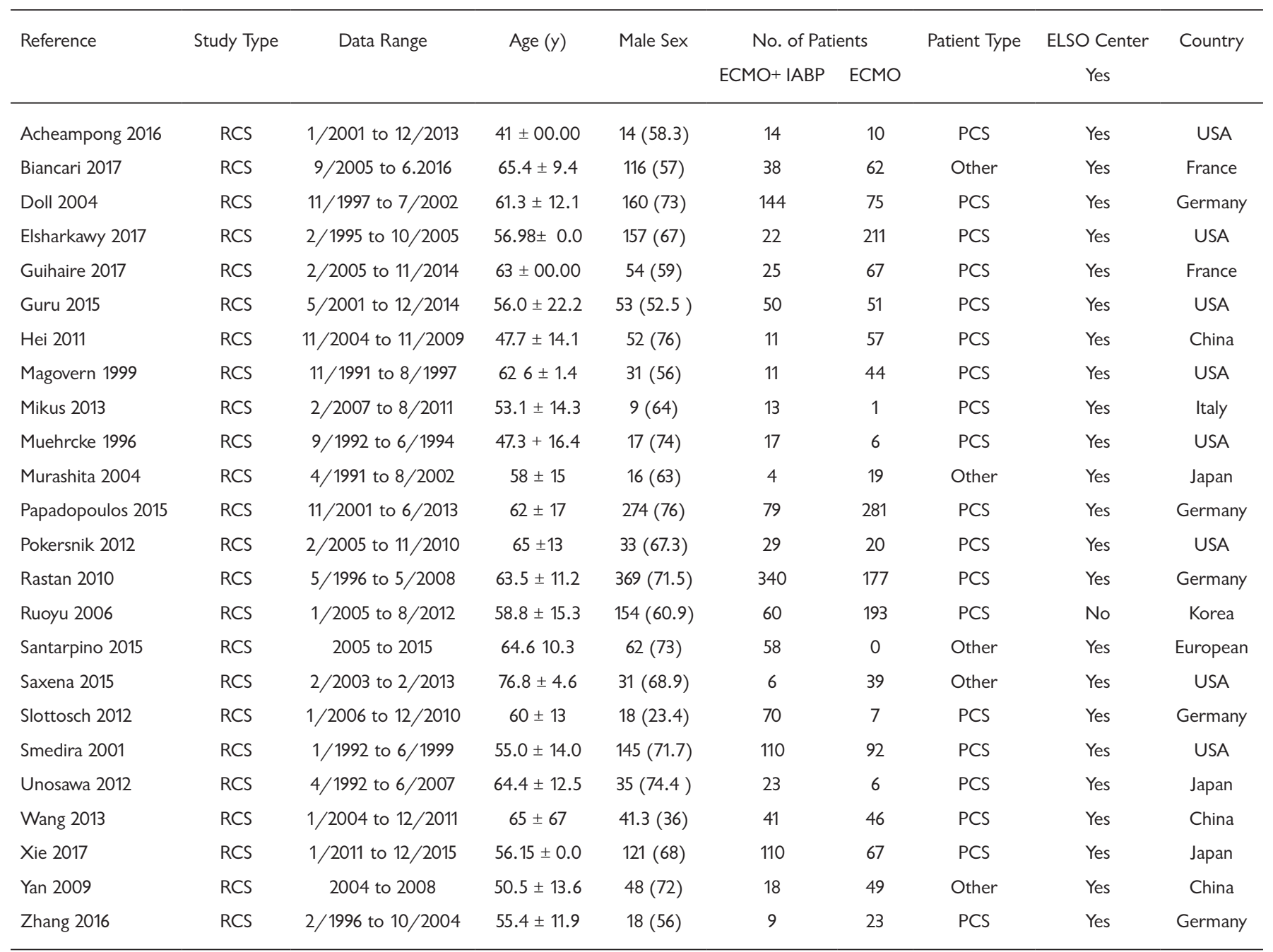

Data are mean \pm SD or $\mathrm{n}(\%)$ unless noted otherwise.

cardiac surgery. In the comparison between IABP plus ECMO versus ECMO alone with PCS, the analysis confirmed that IABP plus ECMO was associated with significantly decreased in-hospital mortality.

\section{Short-Term MCS after Cardiac Surgery}

The incidence of heart dysfunction with cardiac surgery is as high as $3 \%$ to $5 \%$ among patients receiving routine cardiac surgery procedures, and the majority of those patients can be weaned from $\mathrm{CPB}$ using inotropic drugs or IABP after cardiac surgery [Meani 2018; Doshi 2018]. Hemodynamic deterioration, occurrence of multiorgan dysfunction, and development of the systemic inflammatory response syndrome are reasons for patients with PCS needing ECMO with or without IABP, referred as extracorporeal life support (ELS) [Roland 2010].

Despite the theoretical advantage of MCS devices after cardiac surgery, there is limited high-quality evidence supporting their use. In 2015, a long-term study showed high cost and serious complication rates for postcardiotomy cardiogenic shock in nontransplant cardiothoracic surgery [Khorsandi 2015]. MCS for refractory PCS carries a survival benefit and achieves acceptable functional recovery; however, it still has a higher complication rate [Khorsandi 2016]. Recent large-scale data have demonstrated an increasing trend of MCS use in the management of medical and surgical cardiogenic shock [Agarwal 2015]. Consistent with this literature, our study confirms the most frequent use of ECMO use in cardiogenic shock, in 20 of 24 the studies. ECMO have multiple theoretical advantages with cardiac surgery that include increased cardiac output and coronary and cerebral perfusion, decreased ventricular workload, diastolic augmentation [Pfluecke 2014; Scheidt 1973], rapid bedside access with or without fluoroscopy, high cardiac output support, and robust support of both cardiac and pulmonary function [Brugts 2014]. However, increased left ventricular afterload in ECMO can result in worsening LV performance, increasing pulmonary congestion [Bréchot 2017]. 


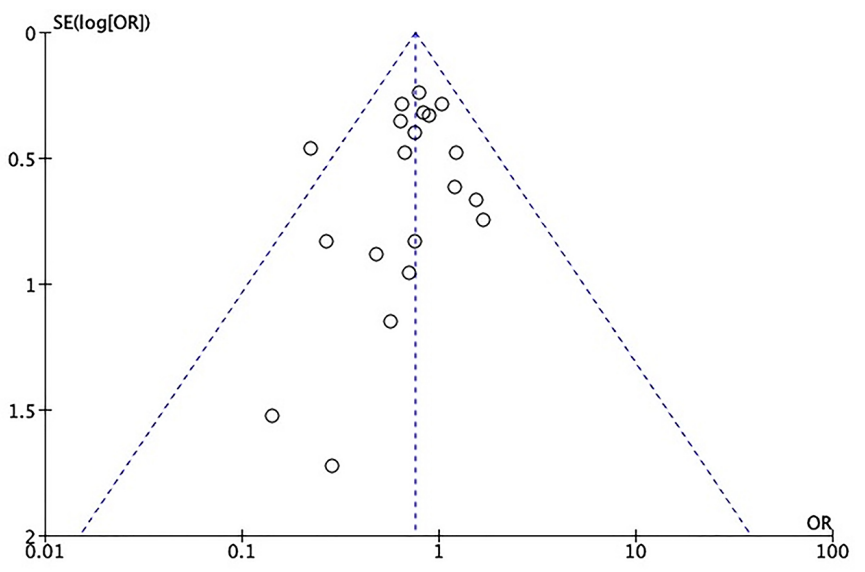

Figure 4. Funnel plot of in-hospital mortality between ECMO plus IABP and ECMO in PCS.

\section{Combined IABP and ECMO after Cardiac Surgery}

The rationale for IABP as a concomitant MCS device after cardiac surgery requiring ECMO is multifold. First, IABP offers the theoretical advantage in decreasing ventricular workload to protect myocardial function and increasing cardiac output to improve distal organ function, especially coronary and cerebral perfusion [Aso 2016]. Importantly, a decrease in left ventricular wall tension, wall stress, and myocardial oxygen demand/consumption [Williams 1982] and improvement in sublingual microcirculatory flow are evident with ECMO [Jung 2008]. Second, ECMO is preferable to IABP because it can provide more robust biventricular support by increasing right ventricular drainage, which can improve gas exchange with the use of bedside equipment [Mosier 2015]. Third, MCS is regarded as a bridge to recovery after cardiac transplantation, and percutaneous MCS, such left ventricular assist, has a lower cost and fewer complications. Thus ECMO plus IABP has been regarded as the second MCS device of choice [Aso 2016]. The purpose of ECMO plus IABP in the PCS is to compensate for heart afterload with improved support of biventricular functions and avoid pulmonary failure, especially after surgery. There are also many risks accompanying ECMO plus IABP: for example, preoperative LV systolic dysfunction and advanced age are associated with poor survival rates in patients with PCS [Muller 2016; Erwan 2014]. In cardiac surgery without PCS, clinical outcome were not improved with IABP plus ECMO versus ECMO alone [Pokersnik 2012; Peigh 2015]. ECMO after adult cardiac surgery is associated with a higher risk of in-hospital mortality, and the negative prognostic effect may be explained by the combined impact of cardiac disease and the extent of the surgical procedures, along with any possible concomitant technical complications occurring during surgery [Truby 2015; Carroll 2015].

To our knowledge, this is the first study to report the comparison of IABP plus ECMO with ECMO alone in cardiac surgery patients. Only 3 meta-analyses have mentioned the risk of MCS after cardiac surgery. Li et al [2019] included 12 studies with 925 cases of ECMO plus IABP and 1190 cases of ECMO and found that ECMO combined with IABP was better than ECMO alone in patients with PCS. This result was similar to our study, but intake was insufficient. Khorsandi et al [2017] conducted a meta-analysis of 24 studies of ECMO for PCS. The in-hospital mortality was $69.2 \%$, which was similar to our study; however, they did not compare ECMO plus IABP after cardiac surgery. Wang et al [2018] also found that short-term and midterm survival rates of PCS treated with ECMO were disappointingly low (34.0\%), and complication rates were relatively high. Therefore, the survival rates of patients after surgery were not changed through the use of ECMO associate with IABP.

Recently, ECMO plus IABP has revealed a potential survival benefit in many critical conditions such as PCS [Karl 2014; Yih-Sharng 2008], PCF [Ma 2014], acute cardiomyopathy or acute myocarditis [Mariana 2011], and postcardiac surgery complications [Alan 2014]. A large, prospective, randomized trial showed that ECMO support was associated with higher in-hospital mortality among adult PCS patients with major vascular complications. Observational studies showed that IABP plus ECMO was an independent risk factor for major vascular complications [Yang 2018] and decreased the rate of heart failure [Doshi 2018; Musiał 2015]. Thus, ECMO plus IABP is a viable option for adult heart transplant with severe rejection and refractory cardiogenic shock [Raffa 2019]. What is more, peripheral and central ECMO configurations showed comparable in-hospital survival and risk of bleeding, and continuous veno-venous hemofiltration and blood product transfusion were significantly lower with the peripheral cannulation strategy [Ouyang 2018].

\section{Limitations}

This study's limitations should be acknowledged. First, it was retrospective, and the lack of randomized studies may cause selection bias. The patients with ECMO plus IABP may also have been associated with an increased risk for adverse outcomes. Several studies did not give clear criteria for patient allocation, which may cause bias in patient selection. Second, no studies on the ELS complications of ECMO plus IABP after surgery in our meta-analysis were included. What is more, the included studies were variable in indications, strategy, cannulation methods, and hemodynamic models of ECMO and IABP. In the future, more detail regarding patient characteristics and treatment algorithms across the studies' pooled data is essential. Finally, this study was largely skewed to patients with surgery, so the results may not necessarily be generalizable to patients with bridges to ventricular assistive devices, and the lack of long term follow-up prevents actuary assessment of treatment effects in both groups.

\section{Conclusions}

Our study showed that the use of IABP plus ECMO with PCS was associated with a lower in-hospital mortality rate; however, for patients without PCS, ECMO plus IABP had no significant advantage in survival rate. In the future, multicenter prospective studies or large prospective multicenter registries are needed to provide further insight into the effects of the combined application. 
ACKNOWLEDGMENTS

This work was partly supported by the National Natural Science Foundation of China (grant no. 11902149). This work is also sponsored by a China Postdoctoral Science Foundation Grant (grant no. 2018M640030).

\section{REFERENCES}

Acheampong B, Johnson JN, Stulak JM, et al. 2016. Postcardiotomy ECMO support after high-risk operations in adult congenital heart disease. Congenit Heart Dis 11:751-755.

Agarwal S, Sud K, Martin JM, Menon V. 2015. Trends in the use of mechanical circulatory support devices in patients presenting with ST-segment elevation myocardial infarction. JACC Cardiovasc Interv 8:1772-1774.

Alan G, Chandrasekar P, Aronow WS, et al. 2014. Peripheral venoarterial extracorporeal membrane oxygenation in combination with intra-aortic balloon counterpulsation in patients with cardiovascular compromise. Cardiology 129:137-143.

Altayyar S, Rochwerg B, Alnasser S, et al. 2014. Intra-aortic balloon pump in patients with cardiogenic shock complicating myocardial infarction: A systematic review and meta-analysis of randomized trials (protocol). Syst Rev 3:24.

Ardawan Julian R, Andreas D, Matthias M, et al. 2010. Early and late outcomes of 517 consecutive adult patients treated with extracorporeal membrane oxygenation for refractory postcardiotomy cardiogenic shock. J Thorac Cardiovasc Surg 139:302-311.e301.

Aso S, Matsui H, Fushimi K, Yasunaga H. 2016. The effect of intraaortic balloon pumping under venoarterial extracorporeal membrane oxygenation on mortality of cardiogenic patients: An analysis using a nationwide inpatient database. Crit Care Med 44:1974-1979.

Biancari F, DaléN M, Perrotti A, et al. 2017. Venoarterial extracorporeal membrane oxygenation after coronary artery bypass grafting: Results of a multicenter study. Int J Cardiol 241:109-114.

Bréchot N, Demondion P, Santi F, et al. 2017. Intra-aortic balloon pump protects against hydrostatic pulmonary oedema during peripheral venoarterial-extracorporeal membrane oxygenation. Eur Heart J Acute Cardiovasc Care 7:62-69.

Brugts JJ, Caliskan K. 2014. Short-term mechanical circulatory support by veno-arterial extracorporeal membrane oxygenation in the management of cardiogenic shock and end-stage heart failure. Exp Rev Cardiovasc Ther 12:145-153.

Burkhoff D, Sayer G, Doshi D, Uriel N. 2015. Hemodynamics of mechanical circulatory support. J Am Coll Cardiol 66:2663-2674.

Cashen K, Thiagarajan RR, Collins JW Jr, et al. 2015. Extracorporeal membrane oxygenation in pediatric trisomy 21: 30 years of experience from the Extracorporeal Life Support Organization Registry. J Pediatr 167:403-408.

Cheng R, Hachamovitch R, Makkar R, et al. 2015. Lack of survival benefit found with use of intraaortic balloon pump in extracorporeal membrane oxygenation: A pooled experience of 1517 patients. J Invas Cardiol 64:453-458.

Chung BB, Sayer G, Uriel N. 2017. Mechanical circulatory support devices: Methods to optimize hemodynamics during use. Expert Ref Med Devices 14:343-353.
Committee for Scientific Affairs, Sakata R, Fujii Y, Kuwano H. 2011. Thoracic and cardiovascular surgery in Japan during 2009: Annual report by the Japanese Association for Thoracic Surgery. Gen Thorac Cardiovasc Surg 59:636-667.

Doll N, Kiaii B, Borger M, et al. 2004. Five-year results of 219 consecutive patients treated with extracorporeal membrane oxygenation for refractory postoperative cardiogenic shock. Ann Thorac Surg 77:151-157.

Doshi R, Patel K, Decter D, Gupta R, Meraj P. 2018. Trends in the utilisation and in-hospital mortality associated with short-term mechanical circulatory support for heart failure with reduced ejection fraction. Heart Lung Circ 28:e47-e50.

Elsharkawy HA, Li L, Esa WAS, Sessler DI, Bashour CA. 2010. Outcome in patients who require venoarterial extracorporeal membrane oxygenation support after cardiac surgery. J Cardiothorac Vasc Anesth 24:946-951.

Erwan F, Amedeo A, Hervé C, et al. 2014. Current aspects of extracorporeal membrane oxygenation in a tertiary referral centre: Determinants of survival at follow-up. Eur J Cardiothorac Surg 46:665.

Feilong H, Song L, Jingwen L, et al. 2011. Five-year results of 121 consecutive patients treated with extracorporeal membrane oxygenation at Fu Wai Hospital. Artif Organs 35:572-578.

Gray BW, Haft JW, Hirsch JC, Annich GM, Hirschl RB, Bartlett RH. 2015. Extracorporeal life support: Experience with 2,000 patients. ASAIO J 61:2-7.

Guihaire J, Dang VS, Rouze S, et al. 2017. Clinical outcomes in patients after extracorporeal membrane oxygenation support for postcardiotomy cardiogenic shock: A single-centre experience of 92 cases. Interact Cardiovasc Thorac Surg 25:363-369.

Guru P, Sanghavi D, Seelhammer T, Schears G. 2015. Outcome of patients with refractory cardiorespiratory failure managed by combination ECMO and IABP. Crit Care Med 43(12 suppl 1):45.

Guttendorf J, Boujoukos AJ, Ren D, Rosenzweig MQ, Hravnak M. 2014. Discharge outcome in adults treated with extracorporeal membrane oxygenation. Am J Crit Care 23:365-377.

Higgins JP, Thompson SG, Deeks JJ, Altman DG. 2003. Measuring inconsistency in meta-analyses. BMJ 327:557-560.

Hoy FB, Mueller DK, Geiss DM, et al. 2000. Bridge to recovery for postcardiotomy failure: Is there still a role for centrifugal pumps? Ann Thorac Surg 70:1259-1263.

Ingo S, Oliver L, Elmar K, et al. 2013. Outcomes after peripheral extracorporeal membrane oxygenation therapy for postcardiotomy cardiogenic shock: A single-center experience. J Surg Res 181:e47-e55.

Jaski BE, Ortiz B, Alla KR, et al. 2010. A 20-year experience with urgent percutaneous cardiopulmonary bypass for salvage of potential survivors of refractory cardiovascular collapse. J Thorac Cardiovasc Surg 139:753-757.

Jung C, Ferrari M, Gradinger R, et al. 2008. Evaluation of the microcirculation during extracorporeal membrane-oxygenation. Clin Hemorheol Microcirc 40:311-314.

Karl W, Stephan G, Henning E, Hochman JS. 2014. Mechanical circulatory support in cardiogenic shock. Eur Heart J 35:156-167.

Khorsandi M, Dougherty S, Bouamra O, et al. 2017. Extra-corporeal membrane oxygenation for refractory cardiogenic shock after adult cardiac surgery: A systematic review and meta-analysis. J Cardiothorac Surg $12: 55$. 
Khorsandi M, Dougherty S, Sinclair A, et al. 2016. A 20-year multicentre outcome analysis of salvage mechanical circulatory support for refractory cardiogenic shock after cardiac surgery. J Cardiothorac Surg 11:151.

Khorsandi M, Shaikhrezai K, Prasad S, et al. 2015. Advanced mechanical circulatory support for refractory cardiogenic shock after cardiac surgery: An eleven-year experience in Edinburgh. J Cardiothorac Surg 10(suppl 1):A336.

Ko WJ, Lin CY, Chen RJ, Wang SS, Lin FY, Chen YS. 2002. Extracorporeal membrane oxygenation support for adult postcardiotomy cardiogenic shock. Ann Thorac Surg 73:538-545.

Kyun RS, Joon Bum K, Sung Ho J, Suk Jung C, Cheol Hyun C, Jae Won L. 2014. Extracorporeal life support for cardiogenic shock: Influence of concomitant intra-aortic balloon counterpulsation. Eur J Cardiothorac Surg 46:186-192.

Li Y, Yan S, Gao S, et al. 2019 Effect of an intra-aortic balloon pump with venoarterial extracorporeal membrane oxygenation on mortality of patients with cardiogenic shock: A systematic review and meta-analysis. Eur J Cardiothorac Surg 55:395-404

Liberati A, Altman DG, Tetzlaff J, et al. 2009. The PRISMA statement for reporting systematic reviews and meta-analyses of studies that evaluate health care interventions: Explanation and elaboration. Ann Intern Med 151:W65-W94.

Ma P, Zhang Z, Song T, et al. 2014. Combining ECMO with IABP for the treatment of critically ill adult heart failure patients. Heart Lung Circ 23:363-368

Magovern GJ, Jr., Magovern JA, Benckart DH, et al. 1994. Extracorporeal membrane oxygenation: Preliminary results in patients with postcardiotomy cardiogenic shock. Ann Thorac Surg 57:1462-1468.

Magovern GJ, Simpson KA. 1999. Extracorporeal membrane oxygenation for adult cardiac support: The Allegheny experience. Ann Thorac Surg 68:655-661.

Mariana M, Charles-Edouard L, Pascal L, et al. 2011. Outcomes, longterm quality of life, and psychologic assessment of fulminant myocarditis patients rescued by mechanical circulatory support. Crit Care Med 39:1029-1035.

Mascio CE, Austin EH, Jacobs JP, et al. 2014. Perioperative mechanical circulatory support in children: An analysis of the Society of Thoracic Surgeons Congenital Heart Surgery Database. J Thorac Cardiovasc Surg 147:658-665.

Meani P, Delnoij T, Raffa GM, et al. 2018. Protracted aortic valve closure during peripheral veno-arterial extracorporeal life support: Is intra-aortic balloon pump an effective solution? Perfusion 34:35-41.

Meani P, Gelsomino S, Natour E, et al. 2017. Modalities and effects of left ventricle unloading on extracorporeal life support: A review of the current literature. Eur J Heart Failure 19(suppl 2):84.

Mikus E, Tripodi A, Calvi S, Giglio MD, Cavallucci A, Lamarra M. 2013. CentriMag venoarterial extracorporeal membrane oxygenation support as treatment for patients with refractory postcardiotomy cardiogenic shock. ASAIO J 59:18-23.

Mosier JM, Kelsey M, Raz Y, et al. 2015. Extracorporeal membrane oxygenation (ECMO) for critically ill adults in the emergency department: History, current applications, and future directions. Crit Care 19:431.

Muehrcke DD, Mccarthy PM, Stewart RW, et al. 1996. Extracorporeal membrane oxygenation for postcardiotomy cardiogenic shock. Ann Thorac Surg 61:684-691.

Muller G, Flecher E, Lebreton G, et al. 2016. The ENCOURAGE mortality risk score and analysis of long-term outcomes after VA-ECMO for acute myocardial infarction with cardiogenic shock. Intensive Care Med 42:370-378.

Musiał R, Darocha T, Kosiński S, Stoliński J, Sadowski J, Drwiła R. 2015. Application of V-A ECMO therapies for short-term mechanical circulatory support in patients with cardiogenic shock. Anaesthesiol Intensive Ther 47:324-327.

Ouyang D, Gulati G, Ha R, Banerjee D. 2018. Incidence of temporary mechanical circulatory support prior to heart transplantation and impact on post-transplant outcomes. J Heart Lung Transplant 37:1060-1066.

Papadopoulos N, Marinos S, Ahmad ES, et al. 2015. Risk factors associated with adverse outcome following extracorporeal life support: Analysis from 360 consecutive patients. Perfusion 30:284-290.

Peigh G, Cavarocchi N, Keith SW, Hirose H. 2015. Simple new risk score model for adult cardiac extracorporeal membrane oxygenation. J Surg Res 198:273-279.

Pfluecke C, Christoph M, Kolschmann S, et al. 2014. Intra-aortic balloon pump (IABP) counterpulsation improves cerebral perfusion in patients with decreased left ventricular function. Perfusion 29:511-516.

Pokersnik JA, Buda T, Bashour CA, Gonzalez-Stawinski GV. 2012. Have changes in ECMO technology impacted outcomes in adult patients developing postcardiotomy cardiogenic shock? J Cardiac Surg 27:246-252.

Raffa GM, Kowalewski M, Brodie D, et al. 2019. Meta-analysis of peripheral or central ECMO in postcardiotomy and non-postcardiotomy shock. Ann Thorac Surg 107:311-321.

Roland P, Henning L, Michael S, et al. 2010. Intra-aortic balloon counterpulsation in patients with acute myocardial infarction complicated by cardiogenic shock: The prospective, randomized IABP SHOCK Trial for attenuation of multiorgan dysfunction syndrome. Crit Care Med $38: 152-160$.

Ruoyu Z, Theo K, Hiroyuki K, et al. 2006. Creatine kinase isoenzyme $\mathrm{MB}$ relative index as predictor of mortality on extracorporeal membrane oxygenation support for postcardiotomy cardiogenic shock in adult patients. Eur J Cardiothorac Surg 30:617-620.

Santarpino G, Ruggieri VG, Mariscalco G, et al. 2015. Outcome in patients having salvage coronary artery bypass grafting. Am J Cardiol 116:1193-1198.

Satoshi U, Akira S, Mitsumasa H, et al. 2013. Long-term outcomes of patients undergoing extracorporeal membrane oxygenation for refractory postcardiotomy cardiogenic shock. Surgery Today 43:264-270.

Saxena P, Neal J, Joyce LD, et al. 2015. Extracorporeal membrane oxygenation support in postcardiotomy elderly patients: The Mayo Clinic experience. Ann Thorac Surg 99:2053-2060.

Scheidt S, Wilner G, Mueller H, et al. 1973. Intra-aortic balloon counterpulsation in cardiogenic-shock: Report of a cooperative clinical trial. N Engl J Med 288:979-984.

Smedira NG, Blackstone EH. 2001. Postcardiotomy mechanical support: Risk factors and outcomes. Ann Thorac Surg 71:S60-S66.

Stroup DF, Berlin JA, Morton SC, et al. 2000. Meta-analysis of observational studies in epidemiology: A proposal for reporting. Meta-analysis Of Observational Studies in Epidemiology (MOOSE) group. JAMA 283:2008-2012.

Thiele H, Zeymer U, Neumann FJ, et al. 2013. Intra-aortic balloon counterpulsation in acute myocardial infarction complicated by cardiogenic shock (IABP-SHOCK II): Final 12 month results of a randomised, open-label trial. Lancet 382:1638-1645. 
Thiele H, Zeymer U, Neumann FJ, et al. 2012. Intraaortic balloon support for myocardial infarction with cardiogenic shock. N Engl J Med 367:1287-1296.

Toshifumi M, Kazuhiro E, Tsukasa M, et al. 2015. Outcome of the perioperative use of percutaneous cardiopulmonary support for adult cardiac surgery: Factors affecting hospital mortality. Artif Organs 28:189-195.

Vallabhajosyula S, O'Horo JC, Antharam P, et al. 2018 Concomitant intra-aortic balloon pump use in cardiogenic shock requiring venoarterial extracorporeal membrane oxygenation. Circ Cardiovasc Intervent 11:e006930.

Viechtbauer W. 2010. Conducting meta-Analyses in R with the metafor package. J Stat Softw 36:1-48.

Wang JG, Han J, Jia YX, Zeng W, Hou XT, Meng X. 2013. Outcome of veno-arterial extracorporeal membrane oxygenation for patients undergoing valvular surgery. PloS One 8:e63924.

Wang L, Wang H, Hou X. 2018. Clinical outcomes of adult patients receiving extracorporeal membrane oxygenation for postcardiotomy cardiogenic shock: A systematic review and meta-analysis. J Cardiothorac Vasc Anesth 32:2087-2093.
Williams DO, Korr KS, Gewirtz H, Most AS. 1982. The effect of intraaortic balloon counterpulsation on regional myocardial blood flow and oxygen consumption in the presence of coronary artery stenosis in patients with unstable angina. Circulation 66:593-597.

Xiaolei Y, Shijie J, Xu M, et al. 2010. Acute kidney injury in adult postcardiotomy patients with extracorporeal membrane oxygenation: Evaluation of the RIFLE classification and the Acute Kidney Injury Network criteria. Eur J Cardiothorac Surg 37:334-338.

Xie HX, Yang F, Jiang CJ, et al. 2017. [Predictors of in-hospital mortality in adult postcardiotomy cardiacgenic shock patients successfully weaned from venoarterial extracorporeal membrane oxygenation]. Zhonghua Yi Xue Za Zhi 97:929. In Chinese.

Yang F, Hou D, Wang J, et al. 2018. Vascular complications in adult postcardiotomy cardiogenic shock patients receiving venoarterial extracorporeal membrane oxygenation. Ann Intens Care 8:72.

Yih-Sharng C, Jou-Wei L, Hsi-Yu Y, et al. 2008. Cardiopulmonary resuscitation with assisted extracorporeal life-support versus conventional cardiopulmonary resuscitation in adults with in-hospital cardiac arrest: An observational study and propensity analysis. Lancet 372:554-561. 